\title{
Operations on Hesitant Linguistic terms sets Induced By Archimedean Triangular Norms And Conorms
}

\author{
Zhaoyan Li, Chenfang Zhao, Zheng Pei \\ School of Computer and Software Engineering, Xihua University, \\ Chengdu 610039, Sichuan, China. \\ E-mail: pqyz@263.net
}

Received 23 August 2017

Accepted 16 January 2018

\begin{abstract}
The aim of the paper is to discuss some new operations on hesitant fuzzy linguistic terms sets based on Archimedean $t$-norm and $t$-conorm. The advantage is that the operations on hesitant fuzzy linguistic terms sets are closed, by studying propositions of the operations on hesitant fuzzy linguistic terms sets, scalar-multiplication addition and power multiplication hesitant fuzzy linguistic terms aggregation operators are proposed. An example is presented to illustrate the practicality of the four well-known scalar-multiplication addition and power multiplication hesitant fuzzy linguistic terms aggregation operators, which are also compared with the symbolic aggregation-based method in the example, results show that scalar-multiplication addition and power multiplication hesitant fuzzy linguistic terms aggregation operators can be applied to fuse hesitant fuzzy linguistic terms sets.
\end{abstract}

Keywords: Triangular norms and conorms; 2-tuple linguistic representation; Hesitant fuzzy linguistic terms set; Linguistic aggregation operator; Linguistic decision making.

\section{Introduction}

Group multi-criteria decision making (GMCDM) is to select a satisfying alternative from a group of possible alternatives with respect to multi-criteria. Because various types of uncertainties are in decision making process and the huge amounts of decision information and alternatives are continuously growing ${ }^{5}$, GMCDM is more and more complexity and difficulties in big data. Up to now, many different decision making methods have been proposed to solve various decision making problems ${ }^{1-5}$, in which, because fuzzy linguistic variables provide a more direct way to effectively represent qualitative information in decision making process, linguistic decision makings based on fuzzy linguistic approach have become an important kind of decision makings, intuitively, linguistic decision makings are closest to human being's cognitive processes that occurs in real life and have attracted many scholars to propose linguistic decision making methods ${ }^{6-14}$.

In linguistic decision makings, two common methods to represent linguistic assessments ${ }^{8}$ are: 1) 2-tuple linguistic model ${ }^{7}$, which is composed by linguistic phrases and numerical values in $[-0.5,0.5)$, i.e., let $S_{p}=\left\{s_{0}, \ldots, s_{p}\right\}$ be a initial linguistic term set, for $\beta \in[0, p]$, a 2 -tuple linguistic value corresponding to $\beta$ is $\Delta:[0, p] \longrightarrow S_{p} \times[-0.5,0.5)$, $\Delta(\beta)=s_{\beta}=\left(s_{i}, \alpha\right)$, where, $i=\operatorname{round}(\beta)$ and $\alpha=$ $\beta-i \in[-0.5,0.5)$, round $(\cdot)$ is the usual round operation and the linguistic term $s_{i}$ is mostly close to $\beta$. Conversely, $\Delta^{-1}: S_{p} \times[-0.5,0.5) \longrightarrow[0, p]$ transforms 2-tuple linguistic value $\left(s_{i}, \alpha\right)$ as $\beta=i+\alpha \in$ $[0, p]$. Denote all 2-tuple linguistic values on $S_{p}$ as $H\left(S_{p}\right)=\left\{s_{\alpha} \mid 0 \leqslant \alpha \leqslant p\right\}$, and for any $s_{\beta_{i}}=\left(s_{i}, \alpha_{i}\right)$ 
and $s_{\beta_{j}}=\left(s_{j}, \alpha_{j}\right)$, then $s_{\beta_{i}} \leqslant s_{\beta_{j}}$ if and only if $\beta_{i} \leqslant$ $\beta_{j}$. Based on 2-tuple linguistic model, many linguistic aggregation operators have been proposed to fuse 2-tuple linguistic assessments of decision makers, such as 2-tuple linguistic weighted or ordered weighted aggregation operators and the probabilistic linguistic terms aggregation operators $6,5,15,46 ; 2$ ) The context-free grammar method ${ }^{8}$, a context-free grammar including different kinds of terminal symbols can be used to generate linguistic term set, i.e., the primary terms such as $\{$ low, medium, high\}, hedges such as $\{$ not, little, much, very $\}$, the relations such as \{lower than, between, higher than\}, conjunctions such as $\{$ and, but $\}$ and disjunctions such as $\{$ or $\}$, for example, "higher than medium" generates a linguistic term set $\{$ medium, high $\}$. By considering decision makers hesitate among different linguistic terms, Rodriguez, et $\mathrm{al}^{8}$ proposed hesitant fuzzy linguistic term set (HFLTS) by utilizing context-free grammars to serves as the basis of increasing the flexibility of the elicitation of linguistic information, it provides us different linguistic expressions to represent decision makers' knowledge/preferences in decision making. Formally, a HFLTS on a linguistic term set $S=\left\{s_{0}, s_{1}, \cdots, s_{p}\right\}$ is described as: $H_{S}$ is an ordered finite subset of the consecutive linguistic terms of $S^{8}$. Intuitively, a HFLTS is also $H_{S}=\left\{s \in S \mid s_{i} \leqslant s \leqslant s_{j}\right\}$ for some $i, j \in\{0, \cdots, p\}$ with $i \leqslant j$, here the non-empty HFLTS $H_{S}=\left\{s \in S \mid s_{i} \leqslant s \leqslant s_{j}\right\}$ is denoted by $H_{S}=\left[s_{i}, s_{j}\right]$, in which, if $i=j$, then $H_{S}=\left[s_{i}, s_{j}\right]$ is the singleton $\left\{s_{i}\right\}^{22}$, all HFLTS on $S$ is denoted by $H S=\left\{\left[s_{i}, s_{j}\right] \mid i, j \in\{0, \ldots, p\}\right.$ and $\left.i \leqslant j\right\}$. Basic operations on HFLTS are as follows $\left.{ }^{8}: 1\right)$ Lower bound: $H_{S^{-}}=\min \left(s_{i}\right)=s_{j}, s_{i} \in H_{S}$ and $s_{i} \geqslant s_{j} \forall i$;2) Upper bound: $H_{S^{+}}=\max \left(s_{i}\right)=s_{j}, s_{i} \in H_{S}$ and $\left.s_{i} \leqslant s_{j} \forall i ; 3\right)$ Complement: $H_{S}^{c}=S-H_{S}=\left\{s_{i} \mid s_{i} \in S\right.$ and $\left.s_{i} \not \nsubseteq H_{S}\right\}$; 4) Union: $H_{S}^{1} \cup H_{S}^{2}=\left\{s_{i} \mid s_{i} \in H_{S}^{1}\right.$ or $\left.s_{i} \in H_{S}^{2}\right\}$; 5) Intersection: $H_{S}^{1} \cap H_{S}^{2}=\left\{s_{i} \mid s_{i} \in H_{S}^{1}\right.$ and $\left.s_{i} \in H_{S}^{2}\right\} ; 6$ ) Envelope: $\operatorname{env}\left(H_{S}\right)=\left[H_{S^{-}}, H_{S^{+}}\right]$. After then, many hesitant fuzzy linguistic terms aggregation operators have been proposed for hesitant fuzzy linguistic decision makings $9,10,11,12,13,16-24$.

From the algebraic operational laws point of view, aggregation operators are mainly based on triangular norm and conorm (briefly $t$-norm and $t$ - conorm for short $)^{25}$, i.e., binary operations $[0,1] \times$ $[0,1] \rightarrow[0,1]$ are such that commutativity, associativity, monotonicity and boundary condition, which serve as a natural generalization of the classical conjunction or disjunction in many valued reasoning systems ${ }^{26}$, due to their interesting algebraic and logical properties, various extended forms of $t$-norm and $t$-conorm and applications in fuzzy logics and many practical problems have been studied in ${ }^{27-35}$. The aggregation operators derived from the $t$-norms and $t$-conorms show great advantages in fusing numerical information, such as aggregation operators on intuitionistic fuzzy set based on Archimedean $t$-norm and $t$-conorm ${ }^{36}$, new aggregation operators derived from Hamacher family of $t$-norms ${ }^{37}$, and a family of hesitant fuzzy Hamacher operators for fusing hesitant fuzzy sets ${ }^{21,44}$.

In this paper, we investigate the linguistic hesitant fuzzy aggregation operators derived from Archimedean $t$-norms and $t$-conorms. To do so, we firstly review Archimedean $t$-norms and $s$-norms. Then we introduce the linguistic hesitant fuzzy Archimedean $t$-norms and $s$-norms and discuss their properties. Finally, we propose hesitant fuzzy linguistic terms weighted mean and geometric mean operators to fuse hesitant fuzzy linguistic terms in linguistic decision making. The rest of this paper is structured as follows: In Section 2, basic concepts of Archimedean $t$-norms and $t$-conorms are reviewed briefly; In Section 3, some new operational laws for HFLTSs based on the four Archimedean $t$ norms and $t$-conorms are proposed and their properties are analyzed, then hesitant fuzzy linguistic terms weighted mean and geometric mean operators induced by the new operational laws for HFLTSs are provided; In Section 4, we present an example to illustrate the practicality of hesitant fuzzy linguistic terms weighted mean and geometric mean operators, and compare with the symbolic aggregation-based method; Section 5 concludes the paper.

\section{Preliminaries}

In this section, we briefly review basic concepts of Archimedean $t$-norms and $t$-conorms, and their applications in aggregation operators. 
Table 1. The four Archimedean $t$-norms and $t$-conorms.

\begin{tabular}{llll}
\hline Types & Notations & Formulas & Functions \\
\hline Algebra & $T^{A}(x, y)$ & $x y$ & $\varphi(z)=-\log (z)$ \\
& $S^{A}(x, y)$ & $x+y-x y$ & $\psi(z)=-\log (1-z)$ \\
\hline \multirow{2}{*}{ Einstein } & $T^{E}(x, y)$ & $\frac{x y}{(1+(1-x)(1-y))}$ & $\varphi(z)=\log \left(\frac{2-z}{z}\right)$ \\
& $S^{E}(x, y)$ & $\frac{x+y}{1+x y}$ & $\psi(z)=\log \left(\frac{2-(1-z)}{1-z}\right)$ \\
\hline \multirow{2}{*}{ Hamacher } & $T_{\gamma}^{H}(x, y)(\gamma>0)$ & $\frac{x y}{(r+(1-r)(x+y-x y))}$ & $\varphi(z)=\log \left(\frac{\gamma+(1-\gamma) z}{z}\right)$ \\
& $S_{\gamma}^{H}(x, y)(\gamma>0)$ & $\frac{(x+y-x y-(1-\gamma) x y)}{1-(1-\gamma) x y}$ & $\psi(z)=\log \left(\frac{1-(1-\gamma) z}{1-z}\right)$ \\
\hline Frank & $T_{\gamma}^{F}(x, y)(\gamma>1)$ & $\log _{\gamma}\left(1+\frac{\left(\gamma^{x}-1\right)\left(\gamma^{y}-1\right)}{r-1}\right)$ & $\varphi(z)=\log \left(\frac{\gamma-1}{\gamma^{z}}-1\right)$ \\
& $S_{\gamma}^{F}(x, y)(\gamma>1)$ & $1-\log _{\gamma}\left(1+\frac{\left(\gamma^{1-x}-1\right)\left(\gamma^{1-y}-1\right)}{r-1}\right)$ & $\psi(z)=\log \left(\frac{\gamma-1}{\gamma^{1-z}}-1\right)$ \\
\hline
\end{tabular}

Formally, $t$-norm is a binary operation $T:[0,1] \times$ $[0,1] \rightarrow[0,1]$ such that commutative, associative, monotone and has 1 as neutral element, i.e., for any $x, y, z \in[0,1], 1) T(x, y)=T(y, x)$; 2) $T(x, T(y, z))=$ $T(T(x, y), z)$; 3) $T(x, y) \leqslant T(x, z)$, if $y \leqslant z ; 4)$ $T(x, 1)=x$. $t$-conorm is a binary operation $S$ : $[0,1] \times[0,1] \rightarrow[0,1]$ such that commutative, associative, monotone and has 0 as neutral element, i.e., for any $x, y, z \in[0,1], S$ satisfies 1)-3) and $4^{\prime}$ ) $S(x, 0)=x$. Dual property of $t$-norm and $t$-conorm is that for any $t$-norm $T$, it's $t$-conorm is $S(x, y)=$ $1-T(1-x, 1-y)$ and vice-versa. A $t$-norm is strict Archimedean and continuous if and only if it is obtained from a continuous additive function $\varphi:[0,1] \longrightarrow[0, \infty)$ that is strictly decreasing with $\varphi(1)=0$, i.e.,

$$
T(x, y)=\varphi^{-1}(\varphi(x)+\varphi(y)),
$$

where $\varphi^{-1}$ is the inverse function of $\varphi$ and $\varphi^{-1}(x)=$ $\sup \{z \in[0,1] \mid \varphi(z)>x\}$. Similarly, $t$-conorm is

$$
S(x, y)=\psi^{-1}(\psi(x)+\psi(y)),
$$

where $\psi(x)=\varphi(1-x)$. The four well-known Archimedean $t$-norms and $t$-conorms are shown in Table $1^{43}$, many interesting and important results about Archimedean $t$-norms and $s$-norms have been studied in ${ }^{25-41}$. Here, we focus on two important applications in aggregation operators based on Archimedean $t$-norms and $t$-conorms.

One is aggregation operators on intuitionistic fuzzy sets ${ }^{45}$ proposed by Xia $\mathrm{in}^{42}$, in which, Xia, et al used Archimedean $t$-norms and $t$-conorms to define new operations on two intuitionistic fuzzy sets, i.e., let $\alpha_{i}=\left(\mu_{\alpha_{i}}, v_{\alpha_{i}}\right)(i=1,2,3)$ be three intuitionistic fuzzy sets, where for any $x \in X$, $0 \leqslant \mu_{\alpha_{i}}(x)+v_{\alpha_{i}}(x) \leqslant 1$, then we have: 1) $\alpha_{1} \oplus$ $\alpha_{1}=\left(S\left(\mu_{\alpha_{1}}, \mu_{\alpha_{2}}\right), T\left(v_{\alpha_{1}}, v_{\alpha_{2}}\right)\right)=\left(\psi^{-1}\left(\psi\left(\mu_{\alpha_{1}}\right)+\right.\right.$ $\left.\left.\left.\psi\left(\mu_{\alpha_{2}}\right)\right), \varphi^{-1}\left(\varphi\left(v_{\alpha_{1}}\right)+\varphi\left(v_{\alpha_{2}}\right)\right)\right) ; \quad 2\right) \quad \alpha_{1} \otimes$ $\alpha_{2}=\left(T\left(\mu_{\alpha_{1}}, \mu_{\alpha_{2}}\right), S\left(v_{\alpha_{1}}, v_{\alpha_{2}}\right)\right)=\left(\varphi^{-1}\left(\varphi\left(\mu_{\alpha_{1}}\right)+\right.\right.$ $\left.\left.\varphi\left(\mu_{\alpha_{2}}\right)\right), \psi^{-1}\left(\psi\left(v_{\alpha_{1}}\right)+\psi\left(v_{\alpha_{2}}\right)\right)\right) ;$ 3) $\lambda \alpha_{3}=$ $\left(\psi^{-1}\left(\lambda \psi\left(v_{\alpha_{3}}\right)\right), \varphi^{-1}\left(\lambda \varphi\left(v_{\alpha_{3}}\right)\right)\right)(\lambda>0)$; 4) $\alpha_{3}^{\lambda}=$ $\left(\varphi^{-1}\left(\lambda \varphi\left(v_{\alpha_{3}}\right)\right), \psi^{-1}\left(\lambda \psi\left(v_{\alpha_{3}}\right)\right)\right)(\lambda>0)$. When $\varphi$ and $\psi$ are selected as four functions in Table 1, we can obtain Algebra, Einstein, Hamacher and Frank operations between two intuitionistic fuzzy sets. As pointed out in ${ }^{42}$, these operations have many interesting properties and are a uniform expressions of many existed operations on intuitionistic fuzzy sets. Accordingly, Xia, et al further proposed two kinds of intuitionistic fuzzy aggregation operators, i.e., let $\alpha_{i}=\left(\mu_{\alpha_{i}}, v_{\alpha_{i}}\right)(i=1, \ldots, n)$ be $n$ intuitionistic fuzzy sets and $w=\left(w_{1}, \ldots, w_{n}\right)$ the weight vector of $\alpha_{i}(i=1, \ldots, n)$, we have

$$
\begin{array}{r}
A T S-I F W A\left(\alpha_{1}, \ldots, \alpha_{n}\right)=\oplus_{i=1}^{n} w_{i} \alpha_{i} \\
=\oplus_{i=1}^{n}\left(\psi^{-1}\left(\sum_{i=1}^{n} w_{i} \psi\left(\mu_{\alpha_{i}}\right)\right), \varphi^{-1}\left(\sum_{i=1}^{n} w_{i} \varphi\left(v_{\alpha_{i}}\right)\right)\right), \\
A T S-I F W G\left(\alpha_{1}, \ldots, \alpha_{n}\right)=\otimes_{i=1}^{n} \alpha_{i}^{w_{i}} \\
=\otimes_{i=1}^{n}\left(\varphi^{-1}\left(\sum_{i=1}^{n} w_{i} \varphi\left(\mu_{\alpha_{i}}\right)\right), \psi^{-1}\left(\sum_{i=1}^{n} w_{i} \psi\left(v_{\alpha_{i}}\right)\right)\right) .
\end{array}
$$

Formally, there are many many interesting properties for aggregation operators ATS - IFWA and $A T S-I F W G^{42}$. 
The other is aggregation operators on 2-tuple linguistic information proposed by Tao in $^{43}$, in which, Tao, et al used Archimedean $t$-norms and $t$-conorms to define new operations on two 2-tuple linguistic representations, i.e., let $S_{p}=$ $\left\{s_{0}, \ldots, s_{p}\right\}$ be a initial linguistic term set, for any $s_{\alpha_{1}}, s_{\alpha_{2}}, s_{\alpha_{3}} \in H\left(S_{p}\right)$, we have: 1) Additive operation $s_{\alpha_{1}} \oplus s_{\alpha_{2}}=\Delta\left(p \times \psi^{-1}\left(\psi\left(\frac{\alpha_{1}}{p}\right)+\psi\left(\frac{\alpha_{2}}{p}\right)\right)\right)$; 2) Multiplication $s_{\alpha_{1}} \otimes s_{\alpha_{2}}=\Delta\left(p \times \varphi^{-1}\left(\varphi\left(\frac{\alpha_{1}}{p}\right)+\right.\right.$ $\left.\left.\varphi\left(\frac{\alpha_{2}}{p}\right)\right)\right)$; 3) Scalar-multiplication $\lambda s_{\alpha_{3}}=\Delta(p \times$ $\left.\psi^{-1}\left(\lambda \psi\left(\frac{\alpha_{3}}{p}\right)\right)\right)(\lambda>0)$; 4) Power operation $s_{\alpha_{3}}^{\lambda}=$ $\Delta\left(p \times \varphi^{-1}\left(\lambda \varphi\left(\frac{\alpha_{3}}{p}\right)\right)\right)(\lambda>0)$. Similarly, when $\varphi$ and $\psi$ are selected as four functions in Table 1, we can obtain Algebra, Einstein, Hamacher and Frank operations between two 2-tuple linguistic representations. Tao, et al discussed many interesting properties of these operations on $H\left(S_{p}\right)$ and proposed aggregation operators on 2-tuple linguistic information, i.e., let $s_{\alpha_{i}} \in H\left(S_{p}\right)(i=1, \ldots, n)$ be $n$ 2-tuple linguistic values and $w=\left(w_{1}, \ldots, w_{n}\right)$ the weight vector of $s_{\alpha_{i}}(i=1, \ldots, n)$, then a successive 2-tuple linguistic weighted arithmetic mean (S2TLWAM) is

$$
\begin{array}{r}
\operatorname{S2TLWAM}\left(s_{\alpha_{1}}, \ldots, s_{\alpha_{n}}\right)=\oplus_{i=1}^{n}\left(w_{i} s_{\alpha_{i}}\right) \\
=\Delta\left(p \times \psi^{-1}\left(\sum_{i=1}^{n}\left(w_{i} \psi\left(\frac{\alpha_{i}}{p}\right)\right)\right)\right) .
\end{array}
$$

A successive 2-tuple linguistic weighted geometric mean $(S 2 T L G M)$ is

$$
\begin{gathered}
\operatorname{S2TLGM}\left(s_{\alpha_{1}}, \ldots, s_{\alpha_{n}}\right)=\otimes_{i=1}^{n} s_{\alpha_{i}}^{w_{i}} \\
=\Delta\left(p \times \varphi^{-1}\left(\sum_{i=1}^{n}\left(w_{i} \varphi\left(\frac{\alpha_{3}}{p}\right)\right)\right)\right) .
\end{gathered}
$$

Formally, S2TLWAM and S2TLGM of 2-tuple linguistic values are extensions of many existed aggregation operators of 2-tuple linguistic values ${ }^{43}$.

Inspired by Xia and Tao's works, in the follows, we discuss new operations for HFLTSs via Archimedean $t$-norms and $t$-conorms, formally, compared HFLTSs with intuitionistic fuzzy sets, it can be noticed that the constraint condition is different, i.e., $0 \leqslant \mu_{\alpha_{i}}(x)+v_{\alpha_{i}}(x) \leqslant 1$ is in intuitionistic fuzzy set, however, $i \leqslant j$ is in HFLTS $H_{S}=\left[s_{i}, s_{j}\right]$. In addition, HFLTS $H_{S}=\left[s_{i}, s_{j}\right]$ is a discrete and consecutive linguistic terms set on $S_{p}$, for intuitionistic fuzzy set $\alpha_{i}=\left(\mu_{\alpha_{i}}, v_{\alpha_{i}}\right), \mu_{\alpha_{i}}$ and $v_{\alpha_{i}}$ are generally continuous membership and non-membership function on $X$. Intuitively, 2-tuple linguistic value $s_{\alpha} \in H\left(S_{p}\right)$ can be understood as a special case of HFLTS $H_{S}=\left[s_{i}, s_{j}\right]$, i.e., the singleton $\left\{s_{\alpha}\right\}$ when $i=j$. These differences lead us to obtain new operations for HFLTSs, which can provide more choices for the decision makers in hesitant fuzzy linguistic environment.

\section{Operations for HFLTSs induced by Archimedean $t$-norms and $t$-conorms}

In this section, we induce new operations on HFLTSs according to Archimedean $t$-norms and $t$ conorms and discuss properties of new operations.

\subsection{Operations for HFLTSS}

According to continuous additive function $\varphi:[0,1]$ $\longrightarrow[0, \infty)$ and $\psi:[0,1] \longrightarrow[0, \infty)(\psi(x)=\varphi(1-x))$ of Archimedean $t$-norms and $t$-conorms, we have the following operations on HFLTSs.

Definition 1. For any $H_{1}=\left[s_{\alpha_{1}}, s_{\beta_{1}}\right], H_{2}=$ $\left[s_{\alpha_{2}}, s_{\beta_{2}}\right] \in H S$ and a scalar $\lambda>0$, operations based on Archimedean $t$-norms and $t$-conorms for HFLTSs are defined as:

1. Additive operation: $H_{1} \oplus H_{2}=[\Delta(p \times$ $\left.\varphi^{-1}\left(\varphi\left(\frac{\alpha_{1}}{p}\right)+\varphi\left(\frac{\alpha_{2}}{p}\right)\right)\right), \Delta\left(p \times \psi^{-1}\left(\psi\left(\frac{\beta_{1}}{p}\right)+\right.\right.$ $\left.\left.\left.\psi\left(\frac{\beta_{2}}{p}\right)\right)\right)\right]$;

2. Multiplication: $\quad H_{1} \otimes H_{2}=[\Delta(p \times$ $\left.\varphi^{-1}\left(\varphi\left(\frac{\alpha_{1}}{p}\right)+\varphi\left(\frac{\beta_{1}}{p}\right)+\varphi\left(\frac{\alpha_{2}}{p}\right)+\varphi\left(\frac{\beta_{2}}{p}\right)\right)\right), \Delta(p \times$ $\psi^{-1}\left(\psi\left(\varphi^{-1}\left(\varphi\left(\frac{\beta_{1}}{p}\right)+\varphi\left(\frac{\alpha_{2}}{p}\right)\right)\right)+\psi\left(\varphi^{-1}\left(\varphi\left(\frac{\alpha_{1}}{p}\right)\right.\right.\right.$ $\left.\left.\left.\left.+\varphi\left(\frac{\beta_{2}}{p}\right)\right)\right)\right)\right)$;

3. Scalar-multiplication: $\quad \lambda \odot H_{1}=[\Delta(p \times$ $\left.\left.\psi^{-1}\left(\lambda \psi\left(\frac{\alpha_{1}}{p}\right)\right)\right), \Delta\left(p \times \psi^{-1}\left(\lambda \psi\left(\frac{\beta_{1}}{p}\right)\right)\right)\right]$;

4. Power operation: $H_{1}^{\lambda}=\left[\Delta\left(\begin{array}{ll}p & \times\end{array}\right.\right.$ $\left.\left.\varphi^{-1}\left(\lambda \varphi\left(\frac{\alpha_{1}}{p}\right)\right)\right), \Delta\left(p \times \varphi^{-1}\left(\lambda \varphi\left(\frac{\beta_{1}}{p}\right)\right)\right)\right]$. 
Compared Definition 1 with Xia and Tao's works, operations on two 2-tuple linguistic representations are adopted in Definition 1. Because the constraint condition $\alpha_{i} \leqslant \beta_{i}$ of $\left[s_{\alpha_{i}}, s_{\beta_{i}}\right]$ must be satisfied, in additive operation on $H_{1}=\left[s_{\alpha_{1}}, s_{\beta_{1}}\right]$ and $H_{2}=\left[s_{\alpha_{2}}, s_{\beta_{2}}\right]$, we respectively use multiplication on $s_{\alpha_{1}}$ and $s_{\alpha_{2}}$ and additive operation on $s_{\beta_{1}}$ and $s_{\beta_{2}}$ to obtain $H_{1} \oplus H_{2}$, this is different to Xia's additive operation on two intuitionistic fuzzy sets; In multiplication on $H_{1}=\left[s_{\alpha_{1}}, s_{\beta_{1}}\right]$ and $H_{2}=\left[s_{\alpha_{2}}, s_{\beta_{2}}\right]$, we first obtain multiplication on $s_{\beta_{1}}$ and $s_{\alpha_{2}}$ and multiplication on $s_{\alpha_{1}}$ and $s_{\beta_{2}}$, respectively, then we use multiplication and additive operation on them to obtain $H_{1} \otimes H_{2}$, this is different to Xia's multiplication on two intuitionistic fuzzy sets; We use scalar-multiplication on $s_{\alpha_{1}}$ and $s_{\beta_{1}}$ to obtain $\lambda \odot H_{1}$, and power operation on $s_{\alpha_{1}}$ and $s_{\beta_{1}}$ to obtain $H_{1}^{\lambda}$, these are different to Xia's scalar-multiplication and power operation on two intuitionistic fuzzy sets.

In Definition 1, if $\varphi$ and $\psi$ are selected as Algebra, Einstein, Hamacher and Frank $t$-norms and $t$-conorms, we have the following distinct representations of operations on HFLTSs, i.e., let $H_{1}=\left[s_{i}, s_{j}\right]$ and $H_{2}=\left[s_{k}, s_{l}\right]$ be two HFLTSs on $S=\left\{s_{0}, \cdots, s_{p}\right\}$ and $\lambda>0$ a scalar. Then

Case 1: Algebra $t$-norm and $t$-conorm based operations on HFLTSs are

1. Algebra additive operation: $H_{1} \oplus_{A} H_{2}=$ $\left[\Delta\left(p \times T^{A}\left(\frac{i}{p}, \frac{k}{p}\right)\right), \Delta\left(p \times S^{A}\left(\frac{j}{p}, \frac{l}{p}\right)\right)\right]$,

2. Algebra Multiplication: $H_{1} \otimes_{A} H_{2}=$ $\left[\Delta\left(p \quad \times \quad T^{A}\left(T^{A}\left(\frac{j}{p}, \frac{k}{p}\right), T^{A}\left(\frac{i}{p}, \frac{l}{p}\right)\right)\right), \Delta(p \quad \times\right.$ $\left.\left.S^{A}\left(T^{A}\left(\frac{j}{p}, \frac{k}{p}\right), T^{A}\left(\frac{i}{p}, \frac{l}{p}\right)\right)\right)\right]$,

3. Algebra scalar-multiplication: $\lambda \odot_{A} H_{1}=$ $\left[\Delta\left(p \times\left(1-\left(1-\frac{i}{p}\right)^{\lambda}\right)\right), \Delta\left(p \times\left(1-\left(1-\frac{j}{p}\right)^{\lambda}\right)\right)\right]$,

4. Algebra power operation: $H_{1}^{\lambda}=[\Delta(p \times$ $\left.\left.\left(\frac{i}{p}\right)^{\lambda}\right), \Delta\left(p \times\left(\frac{j}{p}\right)^{\lambda}\right)\right]$.

Case 2: Einstein $t$-norm and $t$-conorm based operations on HFLTSs are

1. Einstein additive operation: $H_{1} \oplus_{E} H_{2}=$ $\left[\Delta\left(p \times T^{E}\left(\frac{i}{p}, \frac{k}{p}\right)\right), \Delta\left(p \times S^{E}\left(\frac{j}{p}, \frac{l}{p}\right)\right)\right]$,
2. Einstein Multiplication: $H_{1} \otimes_{E} H_{2}=$ $\left[\Delta\left(p \quad \times \quad T^{E}\left(T^{E}\left(\frac{j}{p}, \frac{k}{p}\right), T^{E}\left(\frac{i}{p}, \frac{l}{p}\right)\right)\right), \Delta(p \quad \times\right.$ $\left.\left.S^{E}\left(T^{E}\left(\frac{j}{p}, \frac{k}{p}\right), T^{E}\left(\frac{i}{p}, \frac{l}{p}\right)\right)\right)\right]$,

3. Einstein scalar-multiplication: $\lambda \odot_{E} H_{1}=$ $\left[\Delta\left(p \times \frac{(p+i)^{\lambda}-(p-i)^{\lambda}}{(p+i)^{\lambda}+(p-i)^{\lambda}}\right), \Delta\left(p \times \frac{(p+j)^{\lambda}-(p-j)^{\lambda}}{(p+j)^{\lambda}+(p-j)^{\lambda}}\right)\right]$,

4. Einstein power operation: $H_{1}^{\lambda}=[\Delta(p \times$ $\left.\left.\frac{2 i^{\lambda}}{(2 p-i)^{\lambda}+i^{\lambda}}\right), \Delta\left(p \times \frac{2 j^{\lambda}}{(2 p-j)^{\lambda}+j^{\lambda}}\right)\right]$.

Case 3: Hammer $t$-norm and $t$-conorm based operations on HFLTSs are

1. Hammer additive operation: $H_{1} \oplus_{H} H_{2}=$ $\left[\Delta\left(p \times T^{H}\left(\frac{i}{p}, \frac{k}{p}\right)\right), \Delta\left(p \times S^{H}\left(\frac{j}{p}, \frac{l}{p}\right)\right)\right]$,

2. Hammer Multiplication: $H_{1} \otimes_{H} H_{2}=$ $\left[\Delta\left(p \quad \times \quad T^{H}\left(T^{H}\left(\frac{j}{p}, \frac{k}{p}\right), T^{H}\left(\frac{i}{p}, \frac{l}{p}\right)\right)\right), \Delta(p \quad \times\right.$ $\left.\left.S^{H}\left(T^{H}\left(\frac{j}{p}, \frac{k}{p}\right), T^{H}\left(\frac{i}{p}, \frac{l}{p}\right)\right)\right)\right]$,

3. Hammer scalar-multiplication: $\lambda \odot_{H}$ $H_{1}=\left[\Delta\left(p \times \frac{(p+(\gamma-1) i)^{\lambda}-(p-i)^{\lambda}}{(p+(\gamma-1) i)^{\lambda}+(\gamma-1)(p-i)^{\lambda}}\right), \Delta(p \times\right.$ $\left.\left.\frac{(p+(\gamma-1) j)^{\lambda}-(p-j)^{\lambda}}{(p+(\gamma-1) j)^{\lambda}+(\gamma-1)(p-j)^{\lambda}}\right)\right]$,

4. Hammer power operation: $H_{1}^{\lambda}=$ $\left[\Delta\left(p \quad \times \quad \frac{\gamma i^{\lambda}}{(p+(\gamma-1)(p-i))^{\lambda}+(\gamma-1) i^{\lambda}}\right), \Delta(p \quad \times\right.$ $\left.\left.\frac{\gamma j^{\lambda}}{(p+(\gamma-1)(p-j))^{\lambda}+(\gamma-1) j^{\lambda}}\right)\right]$.

Case 4: Frank $t$-norm and $t$-conorm based operations on HFLTSs are

1. Frank additive operation: $H_{1} \oplus_{F} H_{2}=[\Delta(p \times$ $\left.\left.T^{F}\left(\frac{i}{p}, \frac{k}{p}\right)\right), \Delta\left(p \times S^{F}\left(\frac{j}{p}, \frac{l}{p}\right)\right)\right]$,

2. Frank Multiplication: $H_{1} \otimes_{F} H_{2}=$ $\left[\Delta\left(p \quad \times \quad T^{F}\left(T^{F}\left(\frac{j}{p}, \frac{k}{p}\right), T^{F}\left(\frac{i}{p}, \frac{l}{p}\right)\right)\right), \Delta(p \quad \times\right.$ $\left.\left.S^{F}\left(T^{F}\left(\frac{j}{p}, \frac{k}{p}\right), T^{F}\left(\frac{i}{p}, \frac{l}{p}\right)\right)\right)\right]$,

3. Frank scalar-multiplication: $\lambda \odot_{F} H_{1}=$ $\left[\Delta\left(p \times\left(1-\log _{\gamma}\left(1+\frac{\left(\gamma^{1-\frac{i}{p}}-1\right)^{\lambda}}{(\gamma-1)^{\lambda-1}}\right)\right)\right), \Delta(p \times(1-\right.$ $\left.\left.\left.\log _{\gamma}\left(1+\frac{\left(\gamma^{1-\frac{j}{p}}-1\right)^{\lambda}}{(\gamma-1)^{\lambda-1}}\right)\right)\right)\right]$,

4. Frank power operation: $H_{1}^{\lambda}=\left[\Delta\left(p \times \log _{\gamma}(1+\right.\right.$ $\left.\left.\left.\frac{\left(\gamma^{1-\frac{j}{p}}-1\right)^{\lambda}}{(\gamma-1)^{\lambda-1}}\right)\right), \Delta\left(p \times \log _{\gamma}\left(1+\frac{\left(\gamma^{1-\frac{i}{p}}-1\right)^{\lambda}}{(\gamma-1)^{\lambda-1}}\right)\right)\right]$. 
Example 1. Let a linguistic terms set be $S=$ \{nothing $\left(s_{0}\right)$, low $\left(s_{1}\right)$, medium $\left(s_{2}\right)$, high $\left(s_{3}\right)$, perfect $\left.\left(s_{4}\right)\right\}$, two HFLTSs $H_{1}=\left\{s_{1}, s_{2}, s_{3}\right\}=\left[s_{1}, s_{3}\right]$ and $H_{2}=\left\{s_{2}, s_{3}, s_{4}\right\}=\left[s_{2}, s_{4}\right]$. Select $\varphi(z)=$ $-\log (z), \psi(z)=-\log (1-z)$ and $\lambda=2$, then

$H_{1} \oplus H_{2}=\left[\Delta\left(4 \times \varphi^{-1}\left(\varphi\left(\frac{1}{4}\right)+\varphi\left(\frac{2}{4}\right)\right), \Delta(4 \times\right.\right.$ $\left.\psi^{-1}\left(\psi\left(\frac{3}{4}\right)+\psi\left(\frac{4}{4}\right)\right)\right]=\left[s_{0.5}, s_{4}\right]=\left\{s_{0.5}, s_{1}, s_{2}, s_{3}, s_{4}\right\}$,

$H_{1} \otimes H_{2}=\left[\Delta\left(4 \times \varphi^{-1}\left(\varphi\left(\frac{1}{4}\right)+\varphi\left(\frac{3}{4}\right)+\right.\right.\right.$ $\left.\varphi\left(\frac{2}{4}\right)+\varphi\left(\frac{4}{4}\right)\right), \Delta\left(4 \times \psi^{-1}\left(\psi\left(\varphi^{-1}\left(\varphi\left(\frac{3}{4}\right)+\varphi\left(\frac{2}{4}\right)\right)\right)+\right.\right.$ $\left.\left.\psi\left(\varphi^{-1}\left(\varphi\left(\frac{1}{4}\right)+\varphi\left(\frac{4}{4}\right)\right)\right)\right)\right]=\left[s_{0.375}, s_{2.125}\right]=$ $\left\{s_{0.375}, s_{1}, s_{2}, s_{2.125}\right\}$,

$\lambda \odot H_{1}=\left[\Delta\left(4 \times \psi^{-1}\left(2 \times \psi\left(\frac{1}{4}\right)\right)\right), \Delta\left(4 \times \psi^{-1}(2 \times\right.\right.$ $\left.\left.\left.\psi\left(\frac{3}{4}\right)\right)\right)\right]=\left[s_{1.75}, s_{3.75}\right]=\left\{s_{1.75}, s_{2}, s_{3}, s_{3.75}\right\}$,

$H_{1}^{\lambda}=\left[\Delta\left(4 \times \varphi^{-1}\left(2 \times \varphi\left(\frac{1}{4}\right)\right)\right), \Delta\left(4 \times \varphi^{-1}(2 \times\right.\right.$ $\left.\left.\left.\varphi\left(\frac{3}{4}\right)\right)\right)\right]=\left[s_{0.25}, s_{2.25}\right]=\left\{s_{0.25}, s_{1}, s_{2}, s_{2.25}\right\}$.

\subsection{Properties of operations on HFLTSS}

In this subsection, we discuss several properties of operations on HFLTSs defined in Definition 1.

Proposition 1. Let a linguistic term set $S=$ $\left\{s_{0}, \cdots, s_{p}\right\}$. For any HFLTSs $H_{1}=\left[s_{i}, s_{j}\right]$ and $H_{2}=$ $\left[s_{k}, s_{l}\right]$ in $H S$ and $\lambda>0, H_{1} \oplus H_{2}, H_{1} \otimes H_{2}, \lambda \odot H_{1}$ and $H_{1}^{\lambda}$ are in $H S$.

Proof. According to $t$-norm and $t$-conorm, for any $x, y \in[0,1]$, we have $T(x, y) \leqslant T(x, 1)=x$ and $T(y, x) \leqslant T(y, 1)=y$, i.e., $T(x, y) \leqslant \min \{x, y\}$, and $S(x, y) \geqslant S(x, 0)=x$ and $S(y, x) \geqslant S(y, 0)=$ $y$, i.e., $S(x, y) \geqslant \max \{x, y\}$, these meant that for any $x, y \in[0,1], t$-norm and $t$-conorm, we have $T(x, y) \leqslant \min \{x, y\} \leqslant \max \{x, y\} \leqslant S(x, y)$. Hence, for any Archimedean $t$-norms and $t$-conorms, we have $\varphi^{-1}(\varphi(x)+\varphi(y)) \leqslant \min \{x, y\} \leqslant \max \{x, y\} \leqslant$ $\psi^{-1}(\psi(x)+\psi(y))$.

For any HFLTSs $H_{1}=\left[s_{\alpha_{1}}, s_{\beta_{1}}\right], H_{2}=\left[s_{\alpha_{2}}, s_{\beta_{2}}\right] \in$ $H S$, due to $\frac{\alpha_{1}}{p} \leqslant \frac{\beta_{1}}{p}$ and $\frac{\alpha_{2}}{p} \leqslant \frac{\beta_{2}}{p}$, we have $\varphi^{-1}\left(\varphi\left(\frac{\alpha_{1}}{p}\right)+\varphi\left(\frac{\alpha_{2}}{p}\right)\right) \leqslant \varphi^{-1}\left(\varphi\left(\frac{\beta_{1}}{p}\right)+\varphi\left(\frac{\beta_{2}}{p}\right)\right) \leqslant$ $\psi^{-1}\left(\psi\left(\frac{\beta_{1}}{p}\right)+\psi\left(\frac{\beta_{2}}{p}\right)\right)$, hence, $H_{1} \oplus H_{2}$ is such that $\Delta\left(p \times \varphi^{-1}\left(\varphi\left(\frac{\alpha_{1}}{p}\right)+\varphi\left(\frac{\alpha_{2}}{p}\right)\right) \leqslant \Delta\left(p \times \psi^{-1}\left(\psi\left(\frac{\beta_{1}}{p}\right)+\right.\right.\right.$ $\left.\psi\left(\frac{\beta_{2}}{p}\right)\right)$, i.e., $H_{1} \oplus H_{2}$ is in $H S$.

In $H_{1} \otimes H_{2}$, due to $\varphi^{-1}\left(\varphi\left(\frac{\alpha_{1}}{p}\right)+\varphi\left(\frac{\beta_{1}}{p}\right)+\right.$ $\left.\varphi\left(\frac{\alpha_{2}}{p}\right)+\varphi\left(\frac{\beta_{2}}{p}\right)\right)=\varphi^{-1}\left(\varphi\left(\varphi^{-1}\left(\varphi\left(\frac{\alpha_{1}}{p}\right)+\varphi\left(\frac{\beta_{1}}{p}\right)\right)\right)+\right.$ $\left.\varphi\left(\varphi^{-1}\left(\varphi\left(\frac{\alpha_{2}}{p}\right)+\varphi\left(\frac{\beta_{2}}{p}\right)\right)\right)\right) \leqslant \psi^{-1}\left(\psi\left(\varphi^{-1}\left(\varphi\left(\frac{\beta_{1}}{p}\right)+\right.\right.\right.$ $\left.\left.\varphi\left(\frac{\alpha_{2}}{p}\right)\right)\right)+\psi\left(\varphi^{-1}\left(\varphi\left(\frac{\alpha_{1}}{p}\right)+\varphi\left(\frac{\beta_{2}}{p}\right)\right)\right)$, hence, $\Delta(p \times$ $\varphi^{-1}\left(\varphi\left(\frac{\alpha_{1}}{p}\right)+\varphi\left(\frac{\beta_{1}}{p}\right)+\varphi\left(\frac{\alpha_{2}}{p}\right)+\varphi\left(\frac{\beta_{2}}{p}\right)\right) \leqslant \Delta(p \times$ $\psi^{-1}\left(\psi\left(\varphi^{-1}\left(\varphi\left(\frac{\beta_{1}}{p}\right)+\varphi\left(\frac{\alpha_{2}}{p}\right)\right)\right)+\psi\left(\varphi^{-1}\left(\varphi\left(\frac{\alpha_{1}}{p}\right)+\right.\right.\right.$ $\left.\left.\left.\varphi\left(\frac{\beta_{2}}{p}\right)\right)\right)\right)$, i.e., $H_{1} \otimes H_{2}$ is in $H S$.

Due to for any $\lambda>0, \quad \psi^{-1}\left(\lambda \psi\left(\frac{\alpha_{1}}{p}\right)\right) \leqslant$ $\psi^{-1}\left(\lambda \psi\left(\frac{\beta_{1}}{p}\right)\right)$ and $\varphi^{-1}\left(\lambda \varphi\left(\frac{\alpha_{1}}{p}\right)\right) \leqslant \varphi^{-1}\left(\lambda \varphi\left(\frac{\beta_{1}}{p}\right)\right)$, hence, $\Delta\left(p \times \psi^{-1}\left(\lambda \psi\left(\frac{\alpha_{1}}{p}\right)\right)\right) \leqslant \Delta\left(p \times \psi^{-1}\left(\lambda \psi\left(\frac{\beta_{1}}{p}\right)\right)\right)$ and $\Delta\left(p \times \varphi^{-1}\left(\lambda \varphi\left(\frac{\alpha_{1}}{p}\right)\right)\right) \leqslant \Delta\left(p \times \varphi^{-1}\left(\lambda \varphi\left(\frac{\beta_{1}}{p}\right)\right)\right)$, i.e., $\lambda \odot H_{1}$ and $H_{1}^{\lambda}$ are in $H S$.

The proposition means that Additive operation, Multiplication, scalar-multiplication and power operation on HFLTSs induced By Archimedean $t$ norms and $t$-conorms are closed.

Proposition 2. For any HFLTSs $H_{1}=\left[s_{\alpha_{1}}, s_{\beta_{1}}\right]$, $H_{2}=\left[s_{\alpha_{2}}, s_{\beta_{2}}\right]$ and $H_{3}=\left[s_{\alpha_{3}}, s_{\beta_{3}}\right]$ in HS, the following operational laws are held: 1$) H_{1} \oplus H_{2}=H_{2} \oplus H_{1}$; 2) $\left(H_{1} \oplus H_{2}\right) \oplus H_{3}=H_{1} \oplus\left(H_{2} \oplus H_{3}\right)$; 3) $H_{1} \otimes H_{2}=$ $H_{2} \otimes H_{1}$.

According to commutativity and associativity of functions $\varphi$ and $\psi, 1), 2$ ) and 3) can be easily proved.

Example 2. In Example 1, for HFLTSs $H_{1}=$ $\left[s_{1}, s_{3}\right], H_{2}=\left[s_{2}, s_{4}\right]$ and $H_{3}=\left[s_{3}, s_{4}\right]$, we can easily check that $H_{1} \oplus H_{2}=H_{2} \oplus H_{1}$ and $H_{1} \otimes$ $H_{2}=H_{2} \otimes H_{1}$, in addition, $\left(H_{1} \oplus H_{2}\right) \oplus H_{3}=$ $\left[s_{0.5}, s_{4}\right] \oplus\left[s_{3}, s_{4}\right]=\left[s_{0.375}, s_{4}\right]$ and $H_{1} \oplus\left(H_{2} \oplus H_{3}\right)=$ $\left[s_{1}, s_{3}\right] \oplus\left[s_{1.5}, s_{4}\right]=\left[s_{0.375}, s_{4}\right]$, i.e., $\left(H_{1} \oplus H_{2}\right) \oplus$ $H_{3}=H_{1} \oplus\left(H_{2} \oplus H_{3}\right)$. However, $\left(H_{1} \otimes H_{2}\right) \otimes$ $H_{3}=\left[s_{0.375}, s_{2.125}\right] \otimes\left[s_{3}, s_{4}\right]=\left[s_{0.149}, s_{1.82}\right]$ and $H_{1} \otimes$ $\left(H_{2} \otimes H_{3}\right)=\left[s_{1}, s_{3}\right] \otimes\left[s_{1.5}, s_{3.5}\right]=\left[s_{0.246}, s_{3.754}\right]$, i.e., $\left(H_{1} \otimes H_{2}\right) \otimes H_{3} \neq H_{1} \otimes\left(H_{2} \otimes H_{3}\right)$. This means that multiplication on HFLTSs is not associative.

Denote $* \in\{A, E, H, F\}$ and $\lambda>0$, according to Propositions 1 and 2, for any HFLTSs $H_{1}=$ $\left[s_{\alpha_{1}}, s_{\beta_{1}}\right], H_{2}=\left[s_{\alpha_{2}}, s_{\beta_{2}}\right]$ and $H_{3}=\left[s_{\alpha_{3}}, s_{\beta_{3}}\right]$ in $H S$ and $\lambda>0$, we have the following results: 1) $H_{1} \oplus_{*} H_{2}$, $H_{1} \otimes_{*} H_{2}, \lambda \odot_{*} H_{1}$ and $H_{1}^{\lambda}$ are in $H S$; 2) $H_{1} \oplus_{*} H_{2}=$ $H_{2} \oplus_{*} H_{1},\left(H_{1} \oplus_{*} H_{2}\right) \oplus_{*} H_{3}=H_{1} \oplus_{*}\left(H_{2} \oplus_{*} H_{3}\right)$ and $H_{1} \otimes_{*} H_{2}=H_{2} \otimes_{*} H_{1}$. 


\subsection{Aggregation operators on HFLTSS}

Based on operations on HFLTSs induced by Archimedean $t$-norm and $t$-conorm, we can propose two kinds of hesitant fuzzy linguistic aggregation operators to fuse hesitant fuzzy linguistic terms assessments provided by decision makers in linguistic decision makings, formally, two kinds of hesitant fuzzy linguistic aggregation operators are described as follows:

1. Scalar-multiplication addition hesitant fuzzy linguistic terms aggregation operator: For any HFLTSs $H_{1}=\left[s_{\alpha_{1}}, s_{\beta_{1}}\right], \cdots, H_{n}=\left[s_{\alpha_{n}}, s_{\beta_{n}}\right]$ on linguistic term set $S=\left\{s_{0}, \cdots, s_{p}\right\}$ and $\sum_{i=1}^{n} \lambda_{i}=1\left(\forall \lambda_{i} \in[0,1]\right)$, scalar-multiplication addition hesitant fuzzy linguistic terms aggregation operator is

$$
\oplus_{i=1}^{n}\left(\lambda_{i} \odot H_{i}\right)=\left(\lambda_{1} \odot H_{1}\right) \oplus \cdots \oplus\left(\lambda_{n} \odot H_{n}\right) .
$$

2. Power multiplication hesitant fuzzy linguistic terms aggregation operator: For any HFLTSs $H_{1}=\left[s_{\alpha_{1}}, s_{\beta_{1}}\right], \cdots, H_{n}=\left[s_{\alpha_{n}}, s_{\beta_{n}}\right]$ on linguistic term set $S=\left\{s_{0}, \cdots, s_{p}\right\}$ and $\sum_{i=1}^{n} \lambda_{i}=$ $1\left(\forall \lambda_{i} \in[0,1]\right)$, Power multiplication hesitant fuzzy linguistic terms aggregation operator is

$$
\otimes_{i=1}^{n} H_{i}^{\lambda_{i}}=H_{1}^{\lambda_{1}} \otimes \cdots \otimes H_{n}^{\lambda_{n}} .
$$

In real world practices, when $\oplus \in\left\{\oplus_{A}, \oplus_{E}, \oplus_{H}, \oplus_{F}\right\}$, $\odot \in\left\{\odot_{A}, \odot_{E}, \odot_{H}, \odot_{F}\right\}$ and $\otimes \in\left\{\otimes_{A}, \otimes_{E}, \otimes_{H}, \otimes_{F}\right\}$, we can obtain four scalar-multiplication addition hesitant fuzzy linguistic terms aggregation operators and four power multiplication hesitant fuzzy linguistic terms aggregation operator, which can provide more choices for the decision makers to fuse hesitant fuzzy linguistic assessments in linguistic decision makings.

\section{Applications}

In this section, we provide an example to show scalar-multiplication addition hesitant fuzzy linguistic terms aggregation operator and power multiplication hesitant fuzzy linguistic terms aggregation operator used in hesitant fuzzy linguistic decision making problem, the example was in ${ }^{8}$ to carry out hesitant fuzzy linguistic decision making by using the symbolic aggregation-based method. Here, we use scalar-multiplication addition hesitant fuzzy linguistic terms aggregation operator and power multiplication hesitant fuzzy linguistic terms aggregation operator to deal with the example and compare their results with the symbolic aggregation-based method.

Let $X=\left\{x_{1}, x_{2}, x_{3}\right\}$ be a set of alternatives, $C=$ $\left\{c_{1}, c_{2}, c_{3}\right\}$ be a set of criteria defined for each alternative, and $S=\left\{s_{0}:\right.$ nothing, $s_{1}:$ very low, $s_{2}:$ low, $s_{3}:$ medium, $s_{4}:$ high, $s_{5}:$ very high, $s_{6}:$ perfect $\}$ be the linguistic term set that is used by the context-free grammar GH to generate the linguistic expressions. The HFLTS assessments that are provided in such a problem are shown in Table 2. Due to weights of criteria are not used in the symbolic aggregation-based method $^{8}$, here we select weights $\left(\frac{1}{3}, \frac{1}{3}, \frac{1}{3}\right)$ of criteria to compare scalar-multiplication addition hesitant fuzzy linguistic terms aggregation operator and power multiplication hesitant fuzzy linguistic terms aggregation operator with the symbolic aggregationbased method.

Table 2. The HFLTS assessments that are provided for the decision problem.

\begin{tabular}{ccccc}
\cline { 3 - 4 } & & \multicolumn{3}{c}{ Criteria } \\
\cline { 3 - 5 } & $H_{i j}$ & $c_{1}$ & $c_{2}$ & $c_{3}$ \\
\hline \multirow{4}{*}{ Alternatives } & $x_{1}$ & {$\left[s_{1}, s_{3}\right]$} & {$\left[s_{4}, s_{5}\right]$} & {$\left[s_{4}, s_{4}\right]$} \\
& $x_{2}$ & {$\left[s_{2}, s_{3}\right]$} & {$\left[s_{3}, s_{3}\right]$} & {$\left[s_{0}, s_{2}\right]$} \\
& $x_{3}$ & {$\left[s_{4}, s_{6}\right]$} & {$\left[s_{1}, s_{2}\right]$} & {$\left[s_{4}, s_{6}\right]$} \\
\hline
\end{tabular}

Using scalar-multiplication addition hesitant fuzzy linguistic terms aggregation operator and power multiplication hesitant fuzzy linguistic terms aggregation operator to carry out the example, we fix $\oplus=\oplus_{A}, \odot=\odot_{A}$ and $\otimes=\otimes_{A}$, then the assessment of each alternative is

$$
\begin{aligned}
E_{A}\left(x_{1}\right)= & \left(\frac{1}{3} \odot_{A}\left[s_{1}, s_{3}\right]\right) \oplus_{A}\left(\frac{1}{3} \odot_{A}\left[s_{4}, s_{5}\right]\right) \\
& \oplus_{A}\left(\frac{1}{3} \odot_{A}\left[s_{4}, s_{4}\right]\right) \\
\doteq & {\left[s_{0.033}, s_{4.84}\right], } \\
C_{A}\left(x_{1}\right)= & {\left[s_{1}, s_{3}\right]^{\frac{1}{3}} \oplus_{A}\left[s_{4}, s_{5}\right]^{\frac{1}{3}} \oplus_{A}\left[s_{4}, s_{4}\right]^{\frac{1}{3}} } \\
\doteq & {\left[s_{1.4}, s_{4.737}\right] . }
\end{aligned}
$$

Similarly, we can obtain $E_{A}\left(x_{2}\right) \doteq\left[s_{0}, s_{2.7}\right], C_{A}\left(x_{2}\right)$ $\doteq\left[s_{0}, s_{3.47}\right], \quad E_{A}\left(x_{3}\right) \doteq\left[s_{0.033}, s_{6}\right]$ and $C_{A}\left(x_{3}\right) \doteq$ $\left[s_{1.44}, s_{4.87}\right]$. By using the score function and the 
variance function for HFLTS ${ }^{24}$, we can order assessments $E_{A}\left(x_{1}\right), E_{A}\left(x_{2}\right)$ and $E_{A}\left(x_{3}\right)$ (or $C_{A}\left(x_{1}\right)$, $C_{A}\left(x_{2}\right)$ and $\left.C_{A}\left(x_{3}\right)\right)$ to select the best one alternative, i.e., the number of linguistic terms in HFLTSs $E_{A}\left(x_{1}\right), E_{A}\left(x_{2}\right)$ and $E_{A}\left(x_{3}\right)$ (or $C_{A}\left(x_{1}\right), C_{A}\left(x_{2}\right)$ and $\left.C_{A}\left(x_{3}\right)\right)$ are $\sharp E_{A}\left(x_{1}\right)=\sharp\left\{s_{0.033}, s_{1}, s_{2}, s_{3}, s_{4}, s_{4.84}\right\}=$ $6, \sharp E_{A}\left(x_{2}\right)=4$ and $\sharp E_{A}\left(x_{3}\right)=7$ (or $\sharp C_{A}\left(x_{1}\right)=5$, $\sharp C_{A}\left(x_{2}\right)=5$ and $\sharp C_{A}\left(x_{3}\right)=5$ ), then the score functions of $E_{A}\left(x_{1}\right), E_{A}\left(x_{2}\right)$ and $E_{A}\left(x_{3}\right)$ (or $C_{A}\left(x_{1}\right)$, $C_{A}\left(x_{2}\right)$ and $\left.C_{A}\left(x_{3}\right)\right)$ are

$$
\begin{aligned}
\rho\left(E_{A}\left(x_{1}\right)\right) & =s_{\frac{1}{\sharp E_{A}\left(x_{1}\right)}} \Sigma_{s \alpha \in E_{A}\left(x_{1}\right)} \alpha=s_{\frac{0.033+1+2+3+4+4.84}{6}} \\
& \doteq s_{2.479} \\
\rho\left(E_{A}\left(x_{2}\right)\right) & \doteq s_{1.425}, \rho\left(E_{A}\left(x_{3}\right)\right) \doteq s_{3.519} \\
\rho\left(C_{A}\left(x_{1}\right)\right) & \doteq s_{3.027}, \rho\left(C_{A}\left(x_{2}\right)\right) \doteq s_{1.894} \\
\rho\left(C_{A}\left(x_{3}\right)\right) & \doteq s_{3.062} .
\end{aligned}
$$

The variance functions of $E_{A}\left(x_{1}\right), E_{A}\left(x_{2}\right)$ and $E_{A}\left(x_{3}\right)$ (or $C_{A}\left(x_{1}\right), C_{A}\left(x_{2}\right)$ and $C_{A}\left(x_{3}\right)$ ) are

$$
\begin{aligned}
\sigma\left(E_{A}\left(x_{1}\right)\right) & =s_{\frac{1}{\nexists E_{A}\left(x_{1}\right)}} \sqrt{\sum_{s \alpha, s_{\beta} \in E_{A}\left(x_{1}\right)}(\alpha-\beta)^{2}} \\
& \doteq s_{1.607}, \\
\sigma\left(E_{A}\left(x_{2}\right)\right) & \doteq s_{0.68}, \sigma\left(E_{A}\left(x_{3}\right)\right) \doteq s_{1.961} \\
\sigma\left(C_{A}\left(x_{1}\right)\right) & \doteq s_{1.231}, \sigma\left(C_{A}\left(x_{2}\right)\right) \doteq s_{1.273}, \\
\sigma\left(C_{A}\left(x_{3}\right)\right) & \doteq s_{1.258} .
\end{aligned}
$$

Due to $\rho\left(E_{A}\left(x_{3}\right)\right)>\rho\left(E_{A}\left(x_{1}\right)\right)>\rho\left(E_{A}\left(x_{2}\right)\right)$ (or $\left.\rho\left(C_{A}\left(x_{3}\right)\right)>\rho\left(C_{A}\left(x_{1}\right)\right)>\rho\left(C_{A}\left(x_{2}\right)\right)\right)$, we obtain the ordering on alternatives, $x_{3} \succ x_{1} \succ x_{2}$ and the best alternative is $x_{3}$.

Similarly, we can calculate assessments of alternatives when $\oplus \in\left\{\oplus_{E}, \oplus_{H}, \oplus_{F}\right\}, \odot \in\left\{\odot_{E}, \odot_{H}\right.$, $\left.\odot_{F}\right\}$ and $\otimes \in\left\{\otimes_{E}, \otimes_{H}, \otimes_{F}\right\}$, then compute the score functions and the variance functions for HFLTS assessments of alternatives and their ordering, accordingly, we can obtain the best one alternative, all these are shown in Table 3.

$\mathrm{In}^{8}$, Rodríguez used the symbolic aggregationbased method to carry out the example, more detail, the min-upper and max-lower operators are adopted to obtain the core information of each alternative, such as for alternative $x_{1}$, HFLTSs assessments of $x_{1}$ is $H\left(x_{1}\right)=\left\{\left\{s_{1}, s_{2}, s_{3}\right\},\left\{s_{4}, s_{5}\right\},\left\{s_{4}\right\}\right\}$, then the upper bound of $H\left(x_{1}\right)$ is $H^{+}\left(x_{1}\right)=\left\{\max \left\{s_{1}\right.\right.$, $\left.\left.s_{2}, s_{3}\right\}, \max \left\{s_{4}, s_{5}\right\}, \max \left\{s_{4}\right\}\right\}=\left\{s_{3}, s_{5}, s_{4}\right\}$, the lower bound of $H\left(x_{1}\right)$ is $H^{-}\left(x_{1}\right)=\left\{\min \left\{s_{1}, s_{2}, s_{3}\right\}\right.$, $\left.\min \left\{s_{4}, s_{5}\right\}, \min \left\{s_{4}\right\}\right\}=\left\{s_{1}, s_{4}\right\}, H_{\min }^{+}\left(x_{1}\right)=\min$ $H^{+}\left(x_{1}\right)=\min \left\{s_{3}, s_{5}, s_{4}\right\}=s_{3}$ and $H_{\max }^{-}\left(x_{1}\right)=$ $\operatorname{maxH} H^{-}\left(x_{1}\right)=\max \left\{s_{1}, s_{4}\right\}=s_{4}$, the core information of $x_{1}$ is the linguistic interval $\left[\min \left\{H_{\max }^{-}\left(x_{1}\right), H_{\min }^{+}\left(x_{1}\right)\right\}, \max \left\{H_{\max }^{-}\left(x_{1}\right), H_{\min }^{+}\left(x_{1}\right)\right\}\right]$ $=\left[s_{3}, s_{4}\right]$,others are shown in Table 4 .

Table 4. The core information of each alternative.

\begin{tabular}{|l|l|l|l|}
\hline & $H^{+}, H_{\text {min }}^{+}$ & $H^{-}, H_{\max }^{-}$ & linguistic interval \\
\hline$x_{1}$ & $\left\{s_{3}, s_{4}, s_{5}\right\}, s_{3}$ & $\left\{s_{1}, s_{4}\right\}, s_{4}$ & {$\left[s_{3}, s_{4}\right]$} \\
\hline$x_{2}$ & $\left\{s_{3}, s_{2}\right\}, s_{2}$ & $\left\{s_{2}, s_{3}, s_{0}\right\}, s_{3}$ & {$\left[s_{2}, s_{3}\right]$} \\
\hline$x_{3}$ & $\left\{s_{6}, s_{2}\right\}, s_{2}$ & $\left\{s_{4}, s_{1}\right\}, s_{4}$ & {$\left[s_{2}, s_{4}\right]$} \\
\hline
\end{tabular}

Based on the core information of each alternative in Table 4, the binary preference relation between alternatives is built ${ }^{47}$, i.e., let linguistic intervals $I_{1}=\left[x_{j L}, x_{j R}\right]$ over interval $I_{2}=\left[x_{j^{\prime} L}, x_{j^{\prime} R}\right]$, the binary preference relation is $p_{j j^{\prime}}=P\left(I_{1}>I_{2}\right)$, where

$P\left(I_{1}>I_{2}\right)=\frac{\max \left\{x_{j R}-x_{j^{\prime} L}, 0\right\}-\max \left\{x_{j L}-x_{j^{\prime} R}, 0\right\}}{\left(x_{j R}-x_{j L}\right)+\left(x_{j^{\prime} R}-x_{j^{\prime} L}\right)}$,

such as for alternatives $x_{1}$ and $x_{2}, p_{12}=P\left(a_{1}>\right.$ $\left.a_{2}\right)=\frac{\max \{4-2,0\}-\max \{3-3,0\}}{(4-3)+(3-2)}=1$, based on Table 4, the binary preference relation between three alternatives is the following $P$

$$
P=\left[p_{j j^{\prime}}\right]_{3 \times 3}=\left(\begin{array}{ccc}
- & 1 & 0.667 \\
0 & - & 0.333 \\
0.333 & 0.667 & -
\end{array}\right),
$$

and nondominance degrees of three alternatives are $\mathrm{NDD}_{1}=\min \left\{1-p_{21}^{c}, 1-p_{31}^{c}\right\}=\min \{1-$ $\left.\max \left\{p_{21}-p_{12}, 0\right\}, 1-\max \left\{p_{31}-p_{13}\right\}\right\}=\min \{1-$ $\max \{0-1,0\}, 1-\max \{0.333-0.667,0\}\}=1$, $\mathrm{NDD}_{2}=\min \left\{1-\max \left\{p_{12}-p_{21}, 0\right\}, 1-\max \right.$ $\left.\left\{p_{32}-p_{23}\right\}\right\}=\min \{1-\max \{1-0,0\}, 1-$ $\max \{0.667-0.333,0\}\}=0$ and $\mathrm{NDD}_{3}=\min \{1-$ $\left.\max \left\{p_{13}-p_{31}, 0\right\}, 1-\max \left\{p_{23}-p_{32}\right\}\right\}=\min \{1-$ $\max \{0.667-0.333,0\}, 1-\max \{0.333-0.667$, $0\}\}=0.666$. Accordingly, the ordering of three alternatives is $x_{1} \succ x_{3} \succ x_{2}$ and the alternative $x_{1}$ is selected due to $\mathrm{NDD}_{1}=\max \left\{\mathrm{NDD}_{1}, \mathrm{NDD}_{2}, \mathrm{NDD}_{3}\right\}$.

Compared Table 3 with Table 4 , we notice the following results:

1) The symbolic aggregation-based method does not consider weights of criteria, the core informa- 
Table 3. The four scalar-multiplication addition and power multiplication hesitant fuzzy linguistic terms aggregation operators.

\begin{tabular}{|c|c|c|c|c|c|c|}
\hline Types & Addition $\left(E_{*}\right)$ & $(\rho(*), \sigma(*))$ & Ordering & Multiplication $\left(C_{*}\right)$ & $(\rho(*), \sigma(*))$ & Ordering \\
\hline \multirow{3}{*}{ Algebra } & $x_{1}:\left[s_{0.033}, s_{4.84}\right]$ & $\left(s_{2.479}, s_{1.607}\right)$ & \multirow{3}{*}{$x_{3} \succ x_{1} \succ x_{2}$} & $x_{1}:\left[s_{1.4}, s_{4.737}\right]$ & $\left(s_{3.027}, s_{1.231}\right)$ & \multirow{3}{*}{$x_{3} \succ x_{1} \succ x_{2}$} \\
\hline & $x_{2}:\left[s_{0}, s_{2.7}\right]$ & $\left(s_{1.425}, s_{1.021}\right)$ & & $x_{2}:\left[s_{0}, s_{3.47}\right]$ & $\left(s_{1.894}, s_{1.273}\right)$ & \\
\hline & $x_{3}:\left[s_{0.033}, s_{6}\right]$ & $\left(s_{3.004}, s_{1.961}\right)$ & & $x_{3}:\left[s_{1.44}, s_{4.87}\right]$ & $\left(s_{3.062}, s_{1.258}\right)$ & \\
\hline \multirow{3}{*}{ Einstein } & $x_{1}:\left[s_{0.008}, s_{4.15}\right]$ & $\left(s_{2.360}, s_{1.583}\right)$ & \multirow{3}{*}{$x_{3} \succ x_{1} \succ x_{2}$} & $x_{1}:\left[s_{1.251}, s_{5.316}\right]$ & $\left(s_{3.428}, s_{1.49}\right)$ & \multirow{3}{*}{$x_{3} \succ x_{1} \succ x_{2}$} \\
\hline & $x_{2}:\left[s_{0}, s_{1.395}\right]$ & $\left(s_{0.798}, s_{0.587}\right)$ & & $x_{2}:\left[s_{0}, s_{2.3}\right]$ & $\left(s_{1.325}, s_{0.904}\right)$ & \\
\hline & $x_{3}:\left[s_{0.008}, s_{6}\right]$ & $\left(s_{3.001}, s_{1.967}\right)$ & & $x_{3}:\left[s_{2.072}, s_{5.402}\right]$ & $\left(s_{3.895}, s_{1.235}\right)$ & \\
\hline \multirow{4}{*}{$\begin{array}{l}\text { Hamacher } \\
\gamma=0.5\end{array}$} & $x_{1}:\left[s_{0.071}, s_{4.225}\right]$ & $\left(s_{2.383}, s_{1.516}\right)$ & \multirow{3}{*}{$x_{3} \succ x_{1} \succ x_{2}$} & $x_{1}:\left[s_{1.481}, s_{4.622}\right]$ & $\left(s_{3.021}, s_{0.982}\right)$ & \multirow{3}{*}{$x_{1} \succ x_{3} \succ x_{2}$} \\
\hline & $x_{2}:\left[s_{0}, s_{2.71}\right]$ & $\left(s_{1.425}, s_{1.023}\right)$ & & $x_{2}:\left[s_{0}, s_{2.278}\right]$ & $\left(s_{1.32}, s_{0.825}\right)$ & \\
\hline & $x_{3}:\left[s_{0.071}, s_{6}\right]$ & $\left(s_{3.01}, s_{1.965}\right)$ & & $x_{3}:\left[s_{1.439}, s_{4.462}\right]$ & $\left(s_{2.980}, s_{1.146}\right)$ & \\
\hline & $x_{1}:\left[s_{0.017}, s_{4.164}\right]$ & $\left(s_{2.364}, s_{1.518}\right)$ & \multirow{3}{*}{$x_{3} \succ x_{1} \succ x_{2}$} & $x_{1}:\left[s_{0.569}, s_{4.004}\right]$ & $\left(s_{2.429}, s_{1.352}\right)$ & \multirow{3}{*}{$x_{2} \succ x_{3} \succ x_{1}$} \\
\hline Frank & $x_{2}:\left[s_{0}, s_{2.692}\right]$ & $\left(s_{1.423}, s_{1.018}\right)$ & & $x_{2}:\left[s_{2.557}, s_{5.6}\right]$ & $\left(s_{4.031}, s_{1.152}\right)$ & \\
\hline$\gamma=2$ & $x_{3}:\left[s_{0.017}, s_{6}\right]$ & $\left(s_{3.002}, s_{1.981}\right)$ & & $x_{3}:\left[s_{2.538}, s_{5.438}\right]$ & $\left(s_{4.00}, s_{1.114}\right)$ & \\
\hline
\end{tabular}

tion of each alternative is obtained by using the minupper and max-lower operators. Linguistic intervals of three alternatives in Table 3 are obtained by using scalar-multiplication addition hesitant fuzzy linguistic terms aggregation operator and power multiplication hesitant fuzzy linguistic terms aggregation operator, in which, weights of criteria are considered in these operators.

2) Based on the core information of each alternative, the symbolic aggregation-based method adopts the binary preference relation between three alternatives to order alternatives. However, the score functions and the variance functions for HFLTS are adopted in Table 3 to order alternatives. In fact, if we use the score functions and the variance functions for the core information of each alternative in 4 to order alternatives, we obtain the same ordering $x_{1} \succ x_{3} \succ x_{2}$.

3) From the Archimedean $t$-norm and $t$-conorm point of view, power multiplication hesitant fuzzy linguistic terms aggregation operators are more similar to the min-upper and max-lower operators than scalar-multiplication addition hesitant fuzzy linguistic terms aggregation operator, intuitively, power multiplication hesitant fuzzy linguistic terms aggregation operators are used to obtain common information (or the core information) of assessments of each alternative, in fact, Hamacher power multipli- cation hesitant fuzzy linguistic terms aggregation operators $(\gamma=0.5)$ and the symbolic aggregationbased method obtain the same ordering $x_{1} \succ x_{3} \succ$ $x_{2}$. Because Archimedean $t$-norm is less than min and Archimedean $t$-conorm is more than max, scalar-multiplication addition hesitant fuzzy linguistic terms aggregation operators are used to obtain general information of assessments of each alternative, which can be seen from widths of linguistic intervals shown in Table 3.

\section{Conclusion}

In this paper, we have studied a further application of Archimedean $t$-norm and $t$-conorm under hesitant fuzzy linguistic environment, and proposed scalar-multiplication addition hesitant fuzzy linguistic terms aggregation operator and power multiplication hesitant fuzzy linguistic terms aggregation operator, especially, Algebra, Einstein, Hamacher and Frank scalar-multiplication addition hesitant fuzzy linguistic terms aggregation operators and power multiplication hesitant fuzzy linguistic terms aggregation operators are used in the example to fuse hesitant fuzzy linguistic terms sets. By comparing with the symbolic aggregation-based method in the example, we notice that power multiplication hesitant fuzzy linguistic terms aggregation operator can 
be used to obtain the core information of assessments of each alternative, scalar-multiplication addition hesitant fuzzy linguistic terms aggregation operator can be used to obtain general information of assessments of each alternative, which provide more choices to fuse hesitant fuzzy linguistic assessments in linguistic decision makings.

\section{Acknowledgments}

We appreciate Dr.Rodríguez and the reviewers for their valuable suggestions in improving this paper. This work is partially supported by National Nature Science Foundation of China (Grant No. 61372187) and the fund of lab of Security Insurance of Cyberspace, Sichuan Province (SZJJ2016-038) and Sichuan Educational Committee(17ZA0360).

\section{References}

1. J. Lu, G. Zhang, D. Ruan and F. Wu, Multi-objective group decision making. methods, software and applications with fuzzy set techniques, Imperial College Press, 2007.

2. W. Pedrycz, P. Ekel, and R. Parreiras, Fuzzy multicriteria decision-making: Models, methods and applications, John Wiley and Sons, Ltd, 2011.

3. Z. Pei, D. Ruan, Y. Xu and J. Liu, Linguistic values-based intelligent information processing: Theory, methods, and applications, Atlantis Press, 2010.

4. Z.S. Xu, Hesitant fuzzy sets theory, studies in fuzziness and soft computing, Springer, Heildelberg, 2014.

5. L. Martinez, R.M. Rodríguez and F. Herrera, The 2-tuple Linguistic Model-Computing with Words in Decision Making, Springer International Publishing Switzerland, 2015.

6. F. Herrera and L. Martinez, A 2-tuple fuzzy linguistic representation model for computing with words, IEEE Transactions on Fuzzy Systems, 8, 6, (2000)746-752.

7. F. Herrera and E. Herrera-Viedma, Linguistic decision analysis: steps for solving decision problems under linguistic information, Fuzzy Sets and Systems, 115, (2000)67-82.

8. R.M. Rodríguez, L. Martínez and F. Herrera, Hesitant fuzzy linguistic term sets for decision making, IEEE Transactions on Fuzzy Systems, 20, 1, (2012)109-119.

9. R.M. Rodríguez, L. Martínez and F. Herrera, A group decision making model dealing with comparative linguistic expressions based on hesitant fuzzy linguistic term sets, Information Sciences, 241, 1, (2013)28-42.
10. R.M. Rodríguez, B. Bedregal, et al, A position and perspective analysis of hesitant fuzzy sets on information fusion in decision making. Towards high quality progress, Information Fusion, 29, (2016)89-97.

11. R.M. Rodríguez, L. Martínez, V. Torra, Z.S. Xu, F. Herrera, Hesitant fuzzy sets: state of the art and future directions, International Journal of Intelligent Systems, 29, 6, (2014)495-524.

12. R. M. Rodríguez, L. Martínez, An analysis of symbolic linguistic computing models in decision making, International Journal of General Systems, 42, 1, (2013)121-136.

13. R. M. Rodríguez, A. Labella L. Martínez, An overview on fuzzy modelling of complex linguistic preferences in decision making, International Journal of Computational Intelligence Systems, 9, 1, (2016)81-94.

14. V.B.S. Silva and D.C. Morais, A group decisionmaking approach using a method for constructing a linguistic scale, Information Sciences, $\mathbf{2 8 8}$, (2014)423-436.

15. Q. Pang, H. Wang and Z. Xu, Probabilistic linguistic term sets in multi-attribute group decision making, Information Sciences, 369, (2016)128-143.

16. C. Wei, N. Zhao and X. Tang, Operators and comparisons of hesitant fuzzy linguistic term sets, IEEE Transactions on Fuzzy Systems, 22, (2014)575-585.

17. I. Beg and T. Rashid, TOPSIS for hesitant fuzzy linguistic term sets, International Journal of Intelligent Systems, 28, 12, (2013) 1162-1171.

18. H. Liao, Z. Xu and X.J. Zeng, Distance and similarity measures for hesitant fuzzy linguistic term sets and their application in multi-criteria decision making, Information Sciences, 271, (2014) 125-142.

19. H. Liu and R.M. Rodriguez, A fuzzy envelop for hesitant fuzzy linguistic term set and its application to multicriteria decision making, Information Sciences, 258, (2014) 220-238.

20. F. Meng, X. Chen and Q. Zhang, Multi-attribute decision analysis under a linguistic hesitant fuzzy environment, Information Sciences, 267, (2014) 287-305.

21. C.Q. Tan, W.T. Yi and X.H. Chen, Hesitant fuzzy Hamacher aggregation operators for multicriteria decision making, Applied soft Computing, 26, (2015) 325-349.

22. J. Montserrat-Adell, N. Agell, et al, Modeling group assessments by means of hesitant fuzzy linguistic term sets, Journal of Applied Logic (2016), http://dx.doi.org/10.1016/j.jal.2016.11.005.

23. L-W Lee and S-M Chen, Fuzzy decision making based on likelihood-based comparison relations of hesitant fuzzy linguistic term sets and hesitant fuzzy linguistic operators, Information Sciences, 294, (2015) 513-529.

24. H.C. Liao, Z.S. Xu and X.J. Zeng, Hesitant fuzzy lin- 
guistic VIKOR method and its application in qualitative multiple crideria decision making, IEEE Transactions on Fuzzy Systems, 23, 5, (2015) 1343-1355.

25. B. Schweizer and A. Sklar, Probabilistic metric spaces, New York: North Holland, 1983.

26. V. Novák, I. Perfilieva and J. Močkoř, Mathematical principles of fuzzy logic, Kluwer Academic Publishers, 1999.

27. E.P. Klement, Some mathematical aspects of fuzzy sets: Triangular norms, fuzzy logics, and generalized measures, Fuzzy Sets and Systems, 90, (1997) 133140.

28. E.P. Klement, R. Mesiar and E. Pap, Triangular norms. Position paper I: basic analytical and algebraic properties, Fuzzy Sets and Systems, 143, (2004) 5-26.

29. E.P. Klement, R. Mesiar and E. Pap, Triangular norms. Position paper II: general constructions and parameterized families, Fuzzy Sets and Systems, 145, (2004) 411-438.

30. E.P. Klement, R. Mesiar and E. Pap, Triangular norms. Position paper III: continuous $t$-norms, Fuzzy Sets and Systems, 145, (2004) 439-454.

31. E.P. Klement and M. Navara, A survey on different triangular norm-based fuzzy logics, Fuzzy Sets and Systems, 101, (1999) 241-251.

32. J. Fodor and I.J. Rudas, On continuous triangular norms that are migrative, Fuzzy Sets and Systems, 158, (2007) 1692-1697.

33. D. Butnariu, E.P. Klement and S. Zafrany, On triangular norm-based propositional fuzzy logics, Fuzzy Sets and Systems, 69, (1995) 241-255.

34. B.Q. Hu and C.K. Kwong, On type-2 fuzzy sets and their $t$-norm operations, Information sciences, $\mathbf{2 5 5}$, (2014) 58-81.

35. E. Palmeira, B. Bedregal, R. Mesiar and J. Fernandez, A new way to extend $t$-norms, $t$-conorms and negations, Fuzzy sets and systems, 240, (2014) 1-21.

36. G. Beliakov, H. Bustince, et al, On mean operators for
Atanassov's intuitionistic fuzzy sets, Information Sciences, 181, (2011) 1116-1124.

37. M. Oussalah, On the use of Hamacher's $t$-norms family for information aggregation, Information Science, 153, (2003) 107-154.

38. A. Mesiarová-Zemánková, Continuous additive generators of continuous, conditionally cancellative triangular subnorms, Information Sciences, 339, (2016) 53-63.

39. G. Beliakov, A. Pradera and T. Calvo (Eds.), Aggregation Functions: A Guide for Practitioners, Studies in Fuzziness and Soft Computing, vol. 221, SpringerVerlag, Berlin, Heidelberg, 2007.

40. E.P. Klement, R. Mesiar and E. Pap, Triangular Norms, (Kluwer Academic Publishers, Dordrecht, 2000).

41. A. Mesiarová, Continuous triangular subnorms, Fuzzy Sets and Systems, 142, (2004) 75-83.

42. M.M. Xia, Z.S. Xu and B. Zhu, Some issues on intuitionistic fuzzy aggregation operators based on Archimedean $t$-conorm and $t$-norm, KnowledgeBased Systems, 31, (2012) 78-88.

43. Z.F. Tao, H.Y. Chen, L.G. Zhou and J.P. Liu, On new operational laws of 2-tuple linguistic information using Archimedean $t$-norm and $s$-norm, KnowledgeBased Systems, 66, (2014) 156-165.

44. V. Torra, Hesitant fuzzy sets, International Journal of Intelligent Systems, 25, (2010) 529-539.

45. K.T. Atanassov, Intuitionistic fuzzy sets, Fuzzy Sets and Systems, 20, (1986) 87-96.

46. Z. Pei, D. Ruan, J. Liu and Y. Xu, A linguistic aggregation operator with three kinds of weights for nuclear safeguards evaluation, Knowledge-Based Systems, 28, (2012) 19-26.

47. Y.M. Wang, J.B. Yang and D.L. Xu, A preference aggregation method through the estimation of utility intervals, Computers \& Operations Research, 32, (2005) 2027-2049. 